\title{
ANNAMARIA CANINO \\ Periodic solutions of quadratic lagrangian systems on $p$-convex sets
}

\author{
Annales de la faculté des sciences de Toulouse $5^{e}$ série, tome 12, \\ $\mathrm{n}^{\mathrm{o}} 1$ (1991), p. 37-60 \\ <http://www.numdam.org/item?id=AFST_1991_5_12_1_37_0>
}

(C) Université Paul Sabatier, 1991, tous droits réservés.

L'accès aux archives de la revue «Annales de la faculté des sciences de Toulouse » (http://picard.ups-tlse.fr/ annales/) implique l'accord avec les conditions générales d'utilisation (http://www.numdam.org/conditions). Toute utilisation commerciale ou impression systématique est constitutive d'une infraction pénale. Toute copie ou impression de ce fichier doit contenir la présente mention de copyright.

\section{Numdam}

Article numérisé dans le cadre du programme Numérisation de documents anciens mathématiques http://www.numdam.org/ 


\title{
Periodic solutions of quadratic Lagrangian systems on $p$-convex sets
}

\author{
Annamaria Canino ${ }^{(1)}$
}

RÉSUMÉ. - On prouve l'existence d'une infinité de solutions périodiques pour un système lagrangien quadratique sur une certaine classe d'ensembles non réguliers, c'est-à-dire les ensembles p-convexes. On emploie des méthodes variationnelles en analyse non linéaire et non régulière.

Abstract. - We prove the existence of infinitely many periodic solutions for a quadratic Lagrangian system on a certain class of non-smooth sets, namely the p-convex sets. We use variational methods in non-smooth nonlinear analysis.

\section{Introduction}

If $M$ is a compact submanifold without boundary in $\mathbb{R}^{n}$ and $N_{x} M$ denotes the normal subspace to $M$ at $x$, the study of the Lagrangian system

$$
\frac{\mathrm{d}}{\mathrm{d} s}\left(\nabla_{v} L\left(s, \gamma, \gamma^{\prime}\right)\right)-\nabla_{q} L\left(s, \gamma, \gamma^{\prime}\right) \in N_{\gamma(s)} M
$$

has been carried out in [1], where the existence of infinitely many periodic solutions $\gamma$ is proved under quite general assumptions.

The corresponding problem on manifolds with boundary has been treated in [18], where the existence of a periodic solution is proved and in [5], where the existence of infinitely many periodic solutions is shown. The feature of unilateral constraints (cf. [6], [7], [14], [15], [16], [17], [18]) is that, even if the manifold $M$ is of class $C^{\infty}$, the corresponding variational problem does not have a smooth structure.

(1) Dipartimento di Matematica, Università della Calabria, 87036-Arcavacata di Rende, CS (Italy) 


\section{Annamaria Canino}

For this reason, it seems to be natural to allow for the set $M$ itself a certain kind of irregularity. The aim of the paper is to treat the case in which $M$ is a $p$-convex set (see Def. 1.3) and $L$ is quadratic with respect to $\gamma^{\prime}$, namely

$$
L(s, q, v)=\frac{1}{2}(a(s, q) v, v)-V(s, q)
$$

The particular case $a \equiv \mathrm{Id}, V \equiv 0$, which leads to the study of geodesics, was already treated in [2] and [3].

The main tools are the techniques of non-smooth nonlinear analysis developed in [8], [9], [10] and [11]. Actually, the main part of the paper, the second section, is devoted to the proof that these techniques can be applied to our situation.

\section{Recalls of non-smooth analysis and the main result}

We will recall some notions of non-smooth analysis as developed in [6], $[7],[8],[9],[11]$.

From now on, $H$ will be a real Hilbert space, $|\cdot|$ and $(\cdot, \cdot)$ its norm and scalar product, respectively.

If $u \in H$ and $r>0$, we set $B(u, r)=\{v \in H|| v-u \mid<r\}$.

Definition 1.1. - (see also [6] and [9]) Let $\Omega$ be an open subset of $H$ and $f: \Omega \rightarrow \mathbb{R} \cup\{+\infty\}$ a map.

We set

$$
D(f)=\{u \in \Omega \mid f(u)<+\infty\}
$$

Let $u$ belong to $D(f)$. The function $f$ is said to be subdifferentiable at $u$ if there exists $\alpha \in H$ such that

$$
\liminf _{v \rightarrow u} \frac{f(v)-f(u)-(\alpha, v-u)}{|v-u|} \geq 0 .
$$

We denote by $\partial^{-} f(u)$ the (possibly empty) set of such $\alpha$ 's and we set

$$
D\left(\partial^{-} f\right)=\left\{u \in D(f) \mid \partial^{-} f(u) \neq \emptyset\right\}
$$

It is easy to check that $\partial^{-} f(u)$ is convex and closed $\forall u \in D(f)$. 
If $u \in D\left(\partial^{-} f\right), \operatorname{grad}^{-} f(u)$ will denote the element of minimal norm of $\partial^{-} f(u)$.

Moreover, let $M$ be a subset of $H$. We denote by $I_{M}$ the function:

$$
I_{M}(u)= \begin{cases}0 & u \in M \\ +\infty & u \in H \backslash M\end{cases}
$$

It is easy to check that $\partial^{-} I_{M}(u)$ is a closed convex cone $\forall u \in M$.

We will call (outward) normal cone to $M$ at $u$ the set $\partial^{-} I_{M}(u)$ and tangent cone to $M$ at $u$ its negative polar $\left(\partial^{-} I_{M}(u)\right)^{-}$, i.e.,

$$
\left(\partial^{-} I_{M}(u)\right)^{-}=\left\{v \in H \mid(v, w) \leq 0, \forall w \in \partial^{-} I_{M}(u)\right\}
$$

Remark 1.2. - Let us suppose that $g: \Omega \rightarrow \mathbb{R}$ is Fréchet differentiable at $u \in \Omega$. Then:

$$
\partial^{-}(f+g)(u) \neq \emptyset \text { if and only if } \partial^{-} f(u) \neq \emptyset
$$

and

$$
\partial^{-}(f+g)(u)=\left\{\alpha+\operatorname{grad} g(u) \mid \alpha \in \partial^{-} f(u)\right\}
$$

Let us introduce the class of $p$-convex sets as defined in [2] and [3]. An other characterization of this class is in [4].

Definition 1.3. - $A$ subset $M$ of $H$ is said to be a $p$-convex set if there exists a continuous function $p: M \rightarrow \mathbb{R}^{+}$such that

$$
(\alpha, v-u) \leq p(u)|\alpha||v-u|^{2}
$$

whenever $u, v \in M$ and $\alpha \in \partial^{-} I_{M}(u)$.

Examples of $p$-convex sets are the following ones:

(1) The $C_{\text {loc }}^{1,1}$-submanifolds (possibly with boundary) of $H$;

(2) The convex subsets of $H$;

(3) The images under a $C_{\text {loc }}^{1,1}$ - diffeomorphism of convex sets;

(4) The subset of $\mathbb{R}^{n}:\left\{x|\max | x_{i} \mid \leq 1, \sum x_{i}^{2} \geq 1\right\}$ (note that it is not included in the classes $(1),(2),(3))$. 


\section{Annamaria Canino}

Now, we can state the main result of the paper.

Let $M$ be a $p$-convex subset of $\mathbb{R}^{n}$ and let $L: \mathbb{R} \times \mathbb{R}^{n} \times \mathbb{R}^{n} \rightarrow \mathbb{R}$ be a Lagrangian of the form

$$
L(s, q, v)=\frac{1}{2}(a(s, q) v, v)-V(s, q)
$$

where $a, V$ are of class $C^{2}$ on $\mathbb{R} \times \mathbb{R}^{n}$ and the matrix $a(s, q)$ is symmetric and positive definite, that is there exists a constant $\nu>0$ such that

$$
(a(s, q) v, v) \geq \nu|v|^{2}, \quad \forall s \in \mathbb{R}, \forall q, v \in \mathbb{R}^{n}
$$

Moreover, let us suppose that $a$ and $V$ are 1-periodic in the first variable:

$$
a(s+1, q)=a(s, q) ; \quad V(s+1, q)=V(s, q)
$$

THEOREM 1.4. - Let us suppose that $M$ is compact, connected and noncontractible in itself and that either

a) $\pi_{1}(M)$ has infinitely many conjugacy classes

or

b) $\pi_{1}(M)$ has a finite number of elements.

Then, there exists a sequence $\left\{\gamma_{h}\right\}_{h} \subset W^{2, \infty}\left(\mathbb{R} ; \mathbb{R}^{n}\right)$ such that $\forall h \in \mathbb{N}$

i) $\gamma_{h}$ is 1-periodic and $\gamma_{h}(s) \in M$

ii) $\frac{\mathrm{d}}{\mathrm{d} s}\left(\nabla_{v} L\left(s, \gamma_{h}, \gamma_{h}^{\prime}\right)\right)-\nabla_{q} L\left(s, \gamma_{h}, \gamma_{h}^{\prime}\right) \in \partial^{-} I_{M}\left(\gamma_{h}\right)$ a.e. in $] 0,1[$

iii) $\lim _{h \rightarrow \infty} \int_{0}^{1} L\left(s, \gamma_{h}, \gamma_{h}^{\prime}\right) \mathrm{d} s=+\infty$.

In order to apply the critical point theory for non-smooth functionals some other notions and results have to be recalled.

Definition 1.5. - Let $\Omega$ be an open subset of $H$ and $f: \Omega \rightarrow \mathbb{R} \cup\{+\infty\}$ a function. A point $u \in D(f)$ si said to be a lower critical point for $f$ if $0 \in \partial^{-} f(u) ; c \in \mathbb{R}$ is said to be a critical value of $f$ if there exists $u \in D(f)$ such that

$$
0 \in \partial^{-} f(u) \text { and } f(u)=c .
$$

Definition 1.6. - (see also [8], [11]) Let $\Omega$ be an open subset of $H . A$ function $f: \Omega \rightarrow \mathbb{R} \cup\{+\infty\}$ is said to have a $\varphi$-monotone subdifferential of order two if there exists a continuous function

$$
\chi: D(f)^{2} \times \mathbb{R}^{2} \rightarrow \mathbb{R}^{+}
$$

such that

$$
(\alpha-\beta, u-v) \geq-\chi(u, v, f(u), f(v))\left(1+|\alpha|^{2}+|\beta|^{2}\right)|u-v|^{2}
$$


Periodic solutions of quadratic Lagrangian systems on $p$-convex sets

whenever

$$
u, v \in D\left(\partial^{-} f\right), \quad \alpha \in \partial^{-} f(u) \quad \text { and } \beta \in \partial^{-} f(v)
$$

The notion of $p$-convex set is actually a particular case of the previous notion. In fact, it turns out (see [2]) that a subset $M$ of $H$ is $p$-convex if and only if $I_{M}$ has a $\varphi$-monotone subdifferential of order two.

TheOREM 1.7. - (see [10]) Let $f: H \rightarrow \mathbb{R} \cup\{+\infty\}$ be a lower semicontinuous function with a $\varphi$-monotone subdifferential of order two. We set

$$
d^{*}(u, v)=|u-v|+|f(u)-f(v)|, \quad \forall u, v \in D(f)
$$

Let us suppose that:

i) $\inf _{H} f>-\infty$

ii) every sequence $\left(u_{h}\right)_{h} \subset D\left(\partial^{-} f\right)$ with $\sup _{h} f\left(u_{h}\right)<+\infty$ and $\lim _{h} \operatorname{grad}^{-} f\left(u_{h}\right)=0$ has a subsequence converging in $H$.

Then, $f$ has at least cat $\left(D(f), d^{*}\right)=+\infty$, then

$$
\sup \left\{f(u) \mid u \in D\left(\partial^{-} f\right), 0 \in \partial^{-} f(u)\right\}=+\infty
$$

Let $M$ be a $p$-convex subset of $H$.

Definition 1.8. - Let us denote by $\widehat{A}$ the set of $u^{\prime} s \in H$ with the two properties:

i) $\delta_{p}(u, M)<1$ where $\delta_{p}(u, M)=\limsup _{\substack{|u-w| \rightarrow d(u, M) \\ w \in M}} 2 p(w)|u-w|$.

ii) $\exists r \geq 0$ such that $M \cap\{v \in H|| v-u \mid \leq r\}$ is closed in $H$ and not empty.

Obviously, $M \subset \widehat{A}$ and:

Proposition 1.9. - (see prop. 2.9 in [2]) Let $M \subset H$ be $p$-convex and locally closed. Then $\widehat{A}$ is open and $\forall u \in \widehat{A}$ there exists one and only one $w \in M$ such that $|u-w|=d(u, M)$.

Moreover, if we set $\pi(u)=w$, then

i) $(u-\pi(u)) \in \partial^{-} I_{M}(\pi(u))$ and $2 p(\pi(u))|u-\pi(u)|<1, \forall u \in \widehat{A}$ 
ii) $\left|\pi\left(u_{1}\right)-\pi\left(u_{2}\right)\right| \leq\left(1-p\left(\pi\left(u_{1}\right)\right)\left|u_{1}-\pi\left(u_{1}\right)\right|+\right.$

$$
\begin{gathered}
\left.-p\left(\pi\left(u_{2}\right)\right)\left|u_{2}-\pi\left(u_{2}\right)\right|\right)^{-1}\left|u_{1}-u_{2}\right|, \\
\forall u_{1}, u_{2} \in \widehat{A}
\end{gathered}
$$

iii) $(t \pi(u)+(1-t) u) \in \widehat{A}, \forall u \in \widehat{A}, \forall t \in[0,1]$.

Remark 1.10. - Let us set $A=\{u \in \widehat{A}|4 p(\pi(u))| u-\pi(u) \mid<1\}$. Then $A$ is an open set containing $M$ and, by proposition $1.9 \mathrm{ii}), \pi: A \rightarrow M$ is Lipschitz continuous of constant two.

Proposition 1.11. - (see prop. 2.2 in [2]) Let $M \subset H$ be p-convex. If $\left\{u_{h}\right\}_{h} \subset M$ is a sequence converging to $u \in M$ and $\left\{\alpha_{h}\right\}_{h} \subset H$ is a sequence converging weakly to $\alpha$ with $\alpha_{h} \in \partial^{-} I_{M}\left(u_{h}\right)$, then $\alpha \in \partial^{-} I_{M}(u)$.

Proposition 1.12. - (see prop. 2.12 in [2]) Let $M \subset H$ be locally closed and $p$-convex. Then

$$
\lim _{t \rightarrow 0^{+}} \frac{\pi(u+t v)-u}{t}=P_{u} v, \quad \forall u \in M \quad \text { and } \quad \forall v \in H
$$

where $P_{u}$ is the projection on the tangent cone to $M$ at $u$.

Proposition 1.13. - Let $M \subset H$ be locally closed and $p$-convex. Let $\left\{u_{h}\right\}_{h}$ be a sequence in $M$ converging to $u \in M$ and let $\tau \in\left(\partial^{-} I_{M}(u)\right)^{-}$. Then

$$
\lim _{h} P_{u_{h}} \tau=\tau
$$

Proof. - Since $\left\{P_{u_{h}} \tau\right\}_{h}$ is bounded, up to a subsequence $\left\{P_{u_{h}} \tau\right\}_{h}$ is weakly convergent to some $\xi \in H$. Since $\left(\tau-P_{u_{h}} \tau\right) \in \partial^{-} I_{M}\left(u_{h}\right)$, by proposition 1.11 we have $(\tau-\xi) \in \partial^{-} I_{M}(u)$. Therefore $(\tau-\xi, \tau) \leq 0$, which implies $|\tau| \leq|\xi|$, hence

$$
\underset{h}{\lim \inf _{h}}\left|P_{u_{h}} \tau\right| \geq|\tau|
$$

From the equality

$$
|\tau|^{2}=\left|P_{u_{h}} \tau\right|^{2}+\left|\tau-P_{u_{h}} \tau\right|^{2}
$$

the thesis follows. 


\section{The variational structure of the problem}

In this section, we want to supply our problem, that is the research of periodic orbits of the considered Lagrangian system, with a variational structure. Our aim is to characterize such periodic orbits as lower critical points of the functional

$$
f: L^{2}\left(0,1 ; \mathbb{R}^{n}\right) \rightarrow \mathbb{R} \cup\{+\infty\}
$$

defined in the following way:

$$
f(\gamma)= \begin{cases}\frac{1}{2} \int_{0}^{1}\left(a(s, \gamma) \gamma^{\prime}, \gamma^{\prime}\right) \mathrm{d} s-\int_{0}^{1} V(s, \gamma) \mathrm{d} s & \gamma \in X \\ +\infty & \gamma \in L^{2}\left(0,1 ; \mathbb{R}^{n}\right) \backslash X\end{cases}
$$

where the space of the admissible paths is:

$$
X=\left\{\gamma \in W^{1,2}\left(0,1 ; \mathbb{R}^{n}\right) \mid \gamma(s) \in M, \gamma(0)=\gamma(1)\right\} .
$$

Since $M$ is compact, we shall assume the function $p$ of definition 1.3 to be constant.

Moreover, if $\gamma \in W^{1,2}\left(0,1 ; \mathbb{R}^{n}\right)$ with $\gamma(s) \in M$ and $\delta \in L^{2}\left(0,1 ; \mathbb{R}^{n}\right)$, we set

$$
\left(P_{\gamma} \delta\right)(s)=P_{\gamma(s)} \delta(s)
$$

where $P_{\gamma(s)}$ is the projection on the tangent cone to $M$ at $\gamma(s)$, according to the scalar product

$$
(u, v)_{s}=(a(s, \gamma(s)) u, v) .
$$

Let us also denote by $\pi_{s}$ the projection on $M$ according to the scalar product $(u, v)_{s}$. By remark 1.8 and the assumptions on $a$, there exists an open set $A$ containing $M$ such that each $\pi_{s}$ is defined on $A$ and is Lipschitz continuous of constant 2 .

Let us begin with a regularity result.

TheOREm 2.1. - Let us take $\gamma \in X$. If $\partial^{-} f(\gamma) \neq \emptyset$ then

$$
\gamma \in W^{2,2}\left(0,1 ; \mathbb{R}^{n}\right), \quad \gamma_{+}^{\prime}(0)=\gamma_{-}^{\prime}(1)
$$




\section{Annamaria Canino}

and

$$
\left|\gamma^{\prime \prime}(s)\right| \leq \text { const }\left(1+|\alpha(s)|+\left|\gamma^{\prime}(s)\right|^{2}\right) \text { a.e. }, \quad \forall \alpha \in \partial^{-} f(\gamma) .
$$

Moreover, if $0 \in \partial^{-} f(\gamma)$ then $\gamma \in W^{2, \infty}\left(0,1 ; \mathbb{R}^{n}\right)$.

For the proof of this theorem, we need some lemmas.

Lемма 2.2. - Let us take $\delta \in W^{1,2}\left(0,1 ; \mathbb{R}^{n}\right)$ and $\gamma \in W^{1,2}\left(0,1 ; \mathbb{R}^{n}\right)$ such that $\gamma(s) \in M, \forall s \in[0,1]$.

Then the following facts hold:

a) $\lim _{t \rightarrow 0^{+}} \frac{\pi_{s}(\gamma+t \delta)-\gamma}{t}=P_{\gamma} \delta, \quad \forall s \in[0,1]$

$$
\limsup _{t \rightarrow 0^{+}}^{\text {and }}\left\|\frac{\pi_{s}(\gamma+t \delta)-\gamma}{t}\right\|_{L^{\infty}}<\infty
$$

b) for every sufficiently small $t>0$, we have

$$
\begin{aligned}
& \pi_{s}(\gamma+t \delta) \in W^{1,2}\left(0,1 ; \mathbb{R}^{n}\right) \\
& \text { and a.e. in }] 0,1[ \\
& \left|\left(\pi_{s}(\gamma+t \delta)\right)^{\prime}\right|_{s} \leq\left|\gamma^{\prime}+t \delta^{\prime}\right|_{s}+\text { const }\left|(\gamma+t \delta)-\pi_{s}(\gamma+t \delta)\right|\left(1+\left|\gamma^{\prime}\right|+t\left|\delta^{\prime}\right|\right) \\
& \text { c) } \lim _{t \rightarrow 0^{+}}\left(\pi_{s}(\gamma+t \delta)\right)^{\prime}=\gamma^{\prime} \text { in } L^{2}\left(0,1 ; \mathbb{R}^{n}\right) .
\end{aligned}
$$

Proof. - a) First of all, let us remark that $P_{\gamma} \delta$ is measurable. By proposition 1.12 , we have

$$
\lim _{t \rightarrow 0^{+}} \frac{\pi_{s}(\gamma+t \delta)-\gamma}{t}=P_{\gamma} \delta
$$

and, also

$$
\left|\frac{\pi_{s}(\gamma+t \delta)-\gamma}{t}\right|_{s} \leq 2\left|\frac{\gamma+t \delta-\gamma}{t}\right|_{s} \leq 2|\delta|_{s}
$$

Then, by (2.2.1), we get

$$
\begin{aligned}
\left|\frac{\pi_{s}(\gamma+t \delta)-\gamma}{t}\right| & \leq \text { const }\left|\frac{\pi_{s}(\gamma+t \delta)-\gamma}{t}\right|_{s} \leq \\
& \leq \text { const } 2|\delta|_{s} \leq \\
& \leq \text { const }|\delta|
\end{aligned}
$$

so, the proof of a) is over. 
Periodic solutions of quadratic Lagrangian systems on $p$-convex sets

b) Let us consider the two scalar products

$$
(u, v)_{s_{i}}=\left(A_{s_{i}} u, v\right)=\left(a\left(s_{i}, \gamma\left(s_{i}\right)\right) u, v\right), \quad i=1,2 .
$$

For every $u \in A$, let $w_{s_{i}}=\pi_{s_{i}} u$ the projection of $u$ according to the scalar product $(u, v)_{s_{i}}, i=1,2$. We want to prove that

$$
\left|w_{s_{1}}-w_{s_{2}}\right| \leq \frac{2}{\nu}\left|u-w_{s_{2}}\right|\left|A_{s_{1}}-A_{s_{2}}\right|
$$

Let us observe that, by proposition $1.9, u-w_{s_{1}} \in \partial_{s_{1}}^{-} I_{M}\left(w_{s_{1}}\right)$, that is

$$
\liminf _{v \rightarrow u, v \in M} \frac{\left(u-w_{s_{1}}, w_{s_{1}}-v\right)_{s_{1}}}{\left|w_{s_{1}}-v\right|_{s_{1}}} \geq 0 .
$$

Passing to the usual metric in $\mathbb{R}^{n},(2.2 .4)$ is equivalent to

$$
\liminf _{v \rightarrow u, v \in M} \frac{\left(A_{s_{1}}(u)-A_{s_{1}}\left(w_{s_{1}}\right), w_{s_{1}}-v\right)}{\left|w_{s_{1}}-v\right|} \geq 0 .
$$

By (2.2.5), it is easy to deduce that

$$
A_{s_{1}}(u)-A_{s_{1}}\left(w_{s_{1}}\right) \in \partial^{-} I_{M}\left(w_{s_{1}}\right) .
$$

Analogously,

$$
A_{s_{2}}(u)-A_{s_{2}}\left(w_{s_{2}}\right) \in \partial^{-} I_{M}\left(w_{s_{2}}\right) .
$$

Since $M$ is a $p$-convex set, we have:

$$
\begin{aligned}
& \left(A_{s_{1}}(u)-A_{s_{1}}\left(w_{s_{1}}\right)-A_{s_{2}}(u)+A_{s_{2}}\left(w_{s_{2}}\right), w_{s_{1}}-w_{s_{2}}\right) \geq \\
& \quad \geq-p\left(\left|A_{s_{1}}(u)-A_{s_{1}}\left(w_{s_{1}}\right)\right|+\left|A_{s_{2}}(u)-A_{s_{2}}\left(w_{s_{2}}\right)\right|\right)\left|w_{s_{1}}-w_{s_{2}}\right|^{2} .
\end{aligned}
$$

On the other hand, we have

$$
\begin{aligned}
& \left(A_{s_{1}}(u)-A_{s_{1}}\left(w_{s_{1}}\right)-A_{s_{2}}(u)+A_{s_{2}}\left(w_{s_{2}}\right), w_{s_{1}}-w_{s_{2}}\right) \leq \\
& \quad \leq\left(A_{s_{1}}(u)-A_{s_{2}}(u)+A_{s_{2}}\left(w_{s_{2}}\right)-A_{s_{1}}\left(w_{s_{2}}\right), w_{s_{1}}-w_{s_{2}}\right)+ \\
& \quad+\left(A_{s_{1}}\left(w_{s_{2}}\right)-A_{s_{1}}\left(w_{s_{1}}\right), w_{s_{1}}-w_{s_{2}}\right) \leq \\
& \quad \leq\left(\left(A_{s_{1}}-A_{s_{2}}\right)\left(u-w_{s_{2}}\right), w_{s_{1}}-w_{s_{2}}\right)-\left|w_{s_{1}}-w_{s_{2}}\right|_{s_{1}}^{2}
\end{aligned}
$$


By (2.2.6) and (2.2.7), we obtain

$$
\begin{aligned}
& \left|w_{s_{1}}-w_{s_{2}}\right|_{s_{1}}^{2}+ \\
& \quad-p\left(\left|A_{s_{1}}(u)-A_{s_{1}}\left(w_{s_{1}}\right)\right|+\left|A_{s_{2}}(u)-A_{s_{2}}\left(w_{s_{2}}\right)\right|\right)\left|w_{s_{1}}-w_{s_{2}}\right|^{2} \leq \\
& \quad \leq\left|u-w_{s_{2}}\right|\left|A_{s_{1}}-A_{s_{2}}\right|\left|w_{s_{1}}-w_{s_{2}}\right| .
\end{aligned}
$$

By hypothesis (1.1), it follows

$$
\left|w_{s_{1}}-w_{s_{2}}\right|_{s_{1}}^{2} \geq \nu\left|w_{s_{1}}-w_{s_{2}}\right|^{2}
$$

and then

$$
\begin{aligned}
& {\left[\nu-p\left(\left|A_{s_{1}}(u)-A_{s_{1}}\left(w_{s_{1}}\right)\right|+\left|A_{s_{2}}(u)-A_{s_{2}}\left(w_{s_{2}}\right)\right|\right)\right]\left|w_{s_{1}}-w_{s_{2}}\right|^{2} \leq} \\
& \quad \leq\left|u-w_{s_{2}}\right|\left|A_{s_{1}}-A_{s_{2}}\right|\left|w_{s_{1}}-w_{s_{2}}\right| .
\end{aligned}
$$

By substituting $A$ with a smaller open set containing $M$, we can assume that

$$
\left[\nu-p\left(\left|A_{s_{1}}(u)-A_{s_{1}}\left(w_{s_{1}}\right)\right|+\left|A_{s_{2}}(u)-A_{s_{2}}\left(w_{s_{2}}\right)\right|\right)\right] \geq \frac{\nu}{2}
$$

hence (2.2.3) follows.

Now, let us consider $\left.s_{1}, s_{2} \in\right] 0,1[$. We have

$$
\begin{aligned}
& \frac{\left|\pi_{s_{1}}(\gamma+t \delta)\left(s_{1}\right)-\pi_{s_{2}}(\gamma+t \delta)\left(s_{2}\right)\right|_{s_{1}}}{\left|s_{2}-s_{1}\right|_{s_{1}}} \leq \\
& \leq \frac{\left|\pi_{s_{1}}(\gamma+t \delta)\left(s_{1}\right)-\pi_{s_{1}}(\gamma+t \delta)\left(s_{2}\right)\right|_{s_{1}}}{\left|s_{2}-s_{1}\right|_{s_{1}}}+ \\
& \quad+\frac{\left|\pi_{s_{1}}(\gamma+t \delta)\left(s_{2}\right)-\pi_{s_{2}}(\gamma+t \delta)\left(s_{2}\right)\right|_{s_{1}}}{\left|s_{2}-s_{1}\right|_{s_{1}}} .
\end{aligned}
$$

By i) and ii) of proposition 1.9, we have

$$
\left|\pi_{s_{1}}(\gamma+t \delta)\left(s_{1}\right)-\pi_{s_{1}}(\gamma+t \delta)\left(s_{2}\right)\right|_{s_{1}} \leq \frac{\left|(\gamma+t \delta)\left(s_{1}\right)-(\gamma+t \delta)\left(s_{2}\right)\right|_{s_{1}}}{\mathfrak{D}}
$$

where

$$
\begin{aligned}
\mathfrak{D}=\left(1-p\left|(\gamma+t \delta)\left(s_{1}\right)-\pi_{s_{1}}(\gamma+t \delta)\left(s_{1}\right)\right|_{s_{1}}+\right. \\
\left.-p\left|(\gamma+t \delta)\left(s_{2}\right)-\pi_{s_{1}}(\gamma+t \delta)\left(s_{2}\right)\right|_{s_{1}}\right) .
\end{aligned}
$$


Hence

$$
\begin{aligned}
& \left|\pi_{s_{1}}(\gamma+t \delta)\left(s_{1}\right)-\pi_{s_{1}}(\gamma+t \delta)\left(s_{2}\right)\right|_{s_{1}} \leq \\
& \quad \leq \text { const }\left(\left|\gamma\left(s_{1}\right)-\gamma\left(s_{2}\right)\right|+t\left|\delta\left(s_{1}\right)-\gamma\left(s_{2}\right)\right|\right) .
\end{aligned}
$$

Moreover, by applying (2.2.3), we get

$$
\begin{aligned}
& \left|\pi_{s_{1}}(\gamma+t \delta)\left(s_{2}\right)-\pi_{s_{2}}(\gamma+t \delta)\left(s_{2}\right)\right|_{s_{1}} \leq \\
& \quad \leq \frac{2}{\nu}\left|(\gamma+t \delta)\left(s_{2}\right)-\pi_{s_{2}}(\gamma+t \delta)\left(s_{2}\right)\right|\left|a\left(s_{1}, \gamma\left(s_{1}\right)\right)-a\left(s_{2}, \gamma\left(s_{2}\right)\right)\right| \leq \\
& \quad \leq \text { const }\left|(\gamma+t \delta)\left(s_{2}\right)-\pi_{s_{2}}(\gamma+t \delta)\left(s_{2}\right)\right|\left(\left|s_{2}-s_{1}\right|+\left|\gamma\left(s_{2}\right)-\gamma\left(s_{1}\right)\right|\right) \leq \\
& \quad \leq \text { const }\left(\left|s_{2}-s_{1}\right|+\left|\gamma\left(s_{2}\right)-\gamma\left(s_{1}\right)\right|\right) .
\end{aligned}
$$

Therefore

$$
\pi_{s}(\gamma+t \delta) \in W^{1,2}\left(0,1 ; \mathbb{R}^{n}\right)
$$

and we have a.e. in $] 0,1[$

$$
\begin{aligned}
& \lim _{s_{2} \rightarrow s_{1}} \frac{\left|\pi_{s_{1}}(\gamma+t \delta)\left(s_{1}\right)-\pi_{s_{1}}(\gamma+t \delta)\left(s_{2}\right)\right|_{s_{1}}}{\left|s_{2}-s_{1}\right|_{s_{1}}} \leq \\
& \leq \frac{\left|(\gamma+t \delta)^{\prime}\left(s_{1}\right)\right|_{s_{1}}}{\left(1-2 p\left|(\gamma+t \delta)\left(s_{1}\right)-\pi_{s_{1}}(\gamma+t \delta)\left(s_{1}\right)\right|_{s_{1}}\right)} \leq \\
& \leq\left|(\gamma+t \delta)^{\prime}\left(s_{1}\right)\right|_{s_{1}}+ \\
& \quad+\text { const }\left|(\gamma+t \delta)\left(s_{1}\right)-\pi_{s_{1}}(\gamma+t \delta)\left(s_{1}\right)\right|\left|(\gamma+t \delta)^{\prime}\left(s_{1}\right)\right|
\end{aligned}
$$

and

$$
\begin{aligned}
& \lim _{s_{2} \rightarrow s_{1}} \frac{\left|\pi_{s_{1}}(\gamma+t \delta)\left(s_{2}\right)-\pi_{s_{2}}(\gamma+t \delta)\left(s_{2}\right)\right|_{s_{1}}}{\left|s_{2}-s_{1}\right|_{s_{1}}} \leq \\
& \leq \text { const } \lim _{s_{2} \rightarrow s_{1}}\left|(\gamma+t \delta)\left(s_{2}\right)-\pi_{s_{2}}(\gamma+t \delta)\left(s_{2}\right)\right| \times \\
& \quad \times \frac{\left(\left|s_{2}-s_{1}\right|+\left|\gamma\left(s_{2}\right)-\gamma\left(s_{1}\right)\right|\right)}{\left|s_{2}-s_{1}\right|} \leq \\
& \leq \text { const }\left|(\gamma+t \delta)\left(s_{1}\right)-\pi_{s_{1}}(\gamma+t \delta)\left(s_{1}\right)\right|\left(1+\left|\gamma^{\prime}\left(s_{1}\right)\right|\right) .
\end{aligned}
$$

Hence we have a.e. in $] 0,1[$

$$
\left|\left(\pi_{s}(\gamma+t \delta)\right)^{\prime}\right|_{s} \leq\left|\gamma^{\prime}+t \delta^{\prime}\right|_{s}+\text { const }\left|(\gamma+t \delta)-\pi_{s}(\gamma+t \delta)\right|\left(1+\left|\gamma^{\prime}\right|+t\left|\delta^{\prime}\right|\right)
$$




\section{Annamaria Canino}

c) In $L^{2}\left(0,1 ; \mathbb{R}^{n}\right)$ we can consider the following scalar product

$$
(\eta, \xi)=\int_{0}^{1} a(s, \gamma(s)) \eta(s) \xi(s) \mathrm{d} s=\int_{0}^{1}(\eta(s), \xi(s))_{s} \mathrm{~d} s .
$$

Since, by a) $\pi_{s}(\gamma+t \delta) \rightarrow \gamma$ in $L^{\infty}$ and $\left(\pi_{s}(\gamma+t \delta)\right)^{\prime}$ is bounded in $L^{2}\left(0,1 ; \mathbb{R}^{n}\right)$ as $t \rightarrow 0$, we have that

$$
\left(\pi_{s}(\gamma+t \delta)\right)^{\prime} \text { weakly converges to } \gamma^{\prime} \text { in } L^{2}\left(0,1 ; \mathbb{R}^{n}\right) .
$$

Moreover

$$
\lim _{t \rightarrow 0^{+}}\left|(\gamma+t \delta)-\pi_{s}(\gamma+t \delta)\right|=0 \text { uniformly on }[0,1],
$$

and by b) we have

$$
\limsup _{t \rightarrow 0} \int_{0}^{1}\left|\left(\pi_{s}(\gamma+t \delta)\right)^{\prime}\right|_{s}^{2} \mathrm{~d} s \leq \int_{0}^{1}\left|\gamma^{\prime}\right|_{s}^{2} \mathrm{~d} s .
$$

Combining (2.2.11) and (2.2.12), we get

$$
\lim _{t \rightarrow 0^{+}}\left(\pi_{s}(\gamma+t \delta)\right)^{\prime}=\gamma^{\prime} \text { in } L^{2}\left(0,1 ; \mathbb{R}^{n}\right) \text {. }
$$

Lemma 2.3. - Let us take $\delta \in W^{1,2}\left(0,1 ; \mathbb{R}^{n}\right)$ and $\gamma \in W^{1,2}\left(0,1 ; \mathbb{R}^{n}\right)$ such that $\gamma(s) \in M, \forall s \in[0,1]$.

Then

$$
\begin{aligned}
\liminf _{t \rightarrow 0^{+}} & \frac{1}{t}\left\{\frac{1}{2} \int_{0}^{1}\left(a(s, \gamma)(\gamma+t \delta)^{\prime},(\gamma+t \delta)^{\prime}\right) \mathrm{d} s+\right. \\
& \left.-\frac{1}{2} \int_{0}^{1}\left(a(s, \gamma)\left(\pi_{s}(\gamma+t \delta)\right)^{\prime},\left(\pi_{s}(\gamma+t \delta)\right)^{\prime}\right) \mathrm{d} s\right\} \geq \\
\geq & -\operatorname{const} \int_{0}^{1}\left|\gamma^{\prime}\right|\left(1+\left|\gamma^{\prime}\right|\right)\left|\delta-P_{\gamma} \delta\right| \mathrm{d} s .
\end{aligned}
$$

Proof. - Let us fix $t>0$ and let us take the path $\gamma+t \delta$. If $t$ is small, $(\gamma+t \delta)(s) \in A, \forall s \in[0,1]$.

By lemma $2.2 \mathrm{~b})$, we have $\pi_{s}(\gamma+t \delta) \in W^{1,2}(0,1 ; M)$ and

$$
\begin{aligned}
& \frac{1}{2} \int_{0}^{1} \frac{1}{t}\left\{\left|(\gamma+t \delta)^{\prime}\right|_{s}^{2}-\left|\left(\pi_{s}(\gamma+t \delta)\right)^{\prime}\right|_{s}^{2}\right\} \mathrm{d} s \geq \\
& \geq \frac{1}{2} \int_{0}^{1} \frac{1}{t}\left\{-\operatorname{const}\left|(\gamma+t \delta)-\pi_{s}(\gamma+t \delta)\right|^{2}\left(1+\left|\gamma^{\prime}\right|+t\left|\delta^{\prime}\right|\right)^{2}+\right. \\
& \left.\quad-\text { const }\left|(\gamma+t \delta)^{\prime}\right|\left|(\gamma+t \delta)-\pi_{s}(\gamma+t \delta)\right|\left(1+\left|\gamma^{\prime}\right|+t\left|\delta^{\prime}\right|\right)\right\} \mathrm{d} s .
\end{aligned}
$$


Combining lemma $2.2 \mathrm{c}$ ) with Lebesgue theorem, we get:

$$
\begin{gathered}
\liminf _{t \rightarrow 0^{+}} \frac{1}{2} \int_{0}^{1} \frac{1}{t}\left\{\left|(\gamma+t \delta)^{\prime}\right|_{s}^{2}-\left|\left(\pi_{s}(\gamma+t \delta)\right)^{\prime}\right|_{s}^{2}\right\} \mathrm{d} s \geq \\
\geq-\operatorname{const} \int_{0}^{1}\left|\gamma^{\prime}\right|\left(1+\left|\gamma^{\prime}\right|\right)\left|\delta-P_{\gamma} \delta\right| \mathrm{d} s . \square
\end{gathered}
$$

LemMA 2.4. - Let us take $\delta \in W^{1,2}\left(0,1 ; \mathbb{R}^{n}\right)$ with $\delta(0)=\delta(1)$ and $\alpha \in \partial^{-} f(\gamma)$. Then

$$
\begin{aligned}
& \int_{0}^{1}\left(a(s, \gamma) \gamma^{\prime}, \delta^{\prime}\right) \mathrm{d} s-\int_{0}^{1}\left(\alpha, P_{\gamma} \delta\right) \mathrm{d} s \geq \\
& \geq-\operatorname{const} \int_{0}^{1}\left|\gamma^{\prime}\right|\left(1+\left|\gamma^{\prime}\right|\right)\left|\delta-P_{\gamma} \delta\right| \mathrm{d} s+ \\
& \quad+\int_{0}^{1}\left(\nabla_{q} V(s, \gamma), P_{\gamma} \delta\right) \mathrm{d} s-\frac{1}{2} \int_{0}^{1}\left(\frac{\partial a(s, \gamma)}{\partial q}\left(P_{\gamma} \delta\right) \gamma^{\prime}, \gamma^{\prime}\right) \mathrm{d} s .
\end{aligned}
$$

Proof. - Let us take $\delta \in W^{1,2}\left(0,1 ; \mathbb{R}^{n}\right)$ with $\delta(0)=\delta(1)$ and $t>0$ small enough that

$$
\pi_{s}(\gamma+t \delta) \in M
$$

Let us observe that, by setting

$$
f_{1}(\gamma)=\frac{1}{2} \int_{0}^{1}\left(a(s, \gamma) \gamma^{\prime}, \gamma^{\prime}\right) \mathrm{d} s
$$

from remark 1.2, we deduce that $\alpha \in \partial^{-} f(\gamma)$ if and only if $\alpha=\tilde{\alpha}-\nabla_{q} V(s, \gamma)$ where $\tilde{\alpha} \in \partial^{-} f_{1}(\gamma)$.

Now, let us take $\alpha \in \partial^{-} f(\gamma)$. By proposition 1.12, we have:

$$
\begin{aligned}
& \int_{0}^{1}\left(a(s, \gamma) \gamma^{\prime}, \delta^{\prime}\right) \mathrm{d} s-\int_{0}^{1}\left(\alpha, P_{\gamma} \delta\right) \mathrm{d} s= \\
& =\lim _{t \rightarrow 0^{+}} \frac{1}{t} \int_{0}^{1}\left\{\frac{1}{2}\left(a(s, \gamma)(\gamma+t \delta)^{\prime},(\gamma+t \delta)^{\prime}\right)-\frac{1}{2}\left(a(s, \gamma) \gamma^{\prime}, \gamma^{\prime}\right)+\right. \\
& \left.\quad-\tilde{\alpha}\left(\pi_{s}(\gamma+t \delta)-\gamma\right)\right\} \mathrm{d} s+\int_{0}^{1}\left(\nabla_{q} V(s, \gamma), P_{\gamma} \delta\right) \mathrm{d} s \geq \\
& \geq \liminf _{t \rightarrow 0^{+}} \frac{1}{t} \int_{0}^{1}\left\{\frac{1}{2}\left(a\left(s, \pi_{s}(\gamma+t \delta)\right)\left(\pi_{s}(\gamma+t \delta)\right)^{\prime},\left(\pi_{s}(\gamma+t \delta)\right)^{\prime}\right)+\right.
\end{aligned}
$$




\section{Annamaria Canino}

$$
\begin{aligned}
& \left.-\frac{1}{2}\left(a(s, \gamma) \gamma^{\prime}, \gamma^{\prime}\right)-\tilde{\alpha}\left(\pi_{s}(\gamma+t \delta)-\gamma\right)\right\} \mathrm{d} s+ \\
& +\liminf _{t \rightarrow 0^{+}} \frac{1}{t} \int_{0}^{1}\left\{\frac{1}{2}\left(a(s, \gamma)\left(\pi_{s}(\gamma+t \delta)\right)^{\prime},\left(\pi_{s}(\gamma+t \delta)\right)^{\prime}\right)+\right. \\
& \left.-\frac{1}{2}\left(a\left(s, \pi_{s}(\gamma+t \delta)\right)\left(\pi_{s}(\gamma+t \delta)\right)^{\prime},\left(\pi_{s}(\gamma+t \delta)\right)^{\prime}\right)\right\} \mathrm{d} s+ \\
& +\liminf _{t \rightarrow 0^{+}} \frac{1}{t} \int_{0}^{1}\left\{\frac{1}{2}\left(a(s, \gamma)(\gamma+t \delta)^{\prime},(\gamma+t \delta)^{\prime}\right)+\right. \\
& \left.-\frac{1}{2}\left(a(s, \gamma)\left(\pi_{s}(\gamma+t \delta)\right)^{\prime},\left(\pi_{s}(\gamma+t \delta)\right)^{\prime}\right)\right\} \mathrm{d} s+ \\
& +\int_{0}^{1}\left(\nabla_{q} V(s, \gamma), P_{\gamma} \delta\right) \mathrm{d} s .
\end{aligned}
$$

Recalling that $\left(\pi_{s}(\gamma+t \delta)-\gamma\right) / t$ is bounded in $L^{2}\left(0,1 ; \mathbb{R}^{n}\right)$, by lemma $2.2 \mathrm{c}$ ), proposition 1.12 and Lebesgue theorem, we get

$$
\begin{aligned}
\liminf _{t \rightarrow 0^{+}} & \frac{1}{t} \int_{0}^{1}\left\{\frac{1}{2}\left(a(s, \gamma)\left(\pi_{s}(\gamma+t \delta)\right)^{\prime},\left(\pi_{s}(\gamma+t \delta)\right)^{\prime}\right)+\right. \\
& \left.-\frac{1}{2}\left(a\left(s, \pi_{s}(\gamma+t \delta)\right)\left(\pi_{s}(\gamma+t \delta)\right)^{\prime},\left(\pi_{s}(\gamma+t \delta)\right)^{\prime}\right)\right\} \mathrm{d} s \geq \\
\geq & -\frac{1}{2} \int_{0}^{1}\left(\frac{\partial a(s, \gamma)}{\partial q}\left(P_{\gamma} \delta\right) \gamma^{\prime}, \gamma^{\prime}\right) \mathrm{d} s .
\end{aligned}
$$

Then, by definition 1.1 and lemma 2.3 , we get the thesis.

LEMmA 2.5. - Let us take $\alpha \in L^{2}\left(0,1 ; \mathbb{R}^{n}\right)$ and $\gamma \in W^{1,2}\left(0,1 ; \mathbb{R}^{n}\right)$ such that $\gamma(s) \in M, \forall s \in[0,1]$. Let us suppose that (2.4.1) holds $\forall \delta \in W^{1,2}\left(0,1 ; \mathbb{R}^{n}\right)$ with $\delta(0)=\delta(1)$.

Then

i) $\gamma \in W^{2,2}\left(0,1 ; \mathbb{R}^{n}\right) ; \quad \gamma_{+}^{\prime}(0)=\gamma_{-}^{\prime}(1)$

ii) $\alpha+\frac{\mathrm{d}}{\mathrm{d} s}\left(a(s, \gamma) \gamma^{\prime}\right)+\nabla_{q} V(s, \gamma)-\frac{1}{2} \frac{\partial a(s, \gamma)}{\partial q}\left(\gamma^{\prime}, \gamma^{\prime}\right) \in \partial^{-} I_{M}(\gamma(s))$ a.e.

iii) $\left|\gamma^{\prime \prime}(s)\right| \leq$ const $\left(1+|\alpha(s)|+\left|\gamma^{\prime}(s)\right|^{2}\right)$ a.e. 
Proof. - Since $\left|\delta-P_{\gamma} \delta\right| \leq|\delta|$, by applying Cauchy-Schwartz inequality to (2.4.1), we obtain $\forall \delta \in W^{1,2}\left(0,1 ; \mathbb{R}^{n}\right)$ with $\delta(0)=\delta(1)$

$$
\begin{aligned}
\int_{0}^{1}\left(a(s, \gamma) \gamma^{\prime}, \delta^{\prime}\right) \mathrm{d} s \geq & -\|\alpha\|_{L^{2}}\|\delta\|_{L^{2}}-\text { const } \int_{0}^{1}\left|\gamma^{\prime}\right|\left|\left(1+\left|\gamma^{\prime}\right|\right)\right||\delta| \mathrm{d} s+ \\
& -\int_{0}^{1}\left|\nabla_{q} V(s, \gamma)\right||\delta| \mathrm{d} s-\frac{1}{2} \int_{0}^{1}\left|\frac{\partial a(s, \gamma)}{\partial q}\right||\delta|\left|\gamma^{\prime}\right|^{2} \mathrm{~d} s
\end{aligned}
$$

and then

$$
\begin{aligned}
\left|\int_{0}^{1}\left(a(s, \gamma) \gamma^{\prime}, \delta^{\prime}\right)\right| \mathrm{d} s \leq & \left(\|\alpha\|_{L^{2}}+\text { const } \int_{0}^{1}\left|\gamma^{\prime}\right| \mathrm{d} s+\right. \\
& \left.+ \text { const } \int_{0}^{1}\left|\gamma^{\prime}\right|^{2} \mathrm{~d} s+\text { const }\right)\|\delta\|_{L^{\infty}} .
\end{aligned}
$$

By (2.5.1), we deduce that $a(s, \gamma) \gamma^{\prime} \in L^{\infty}$ and

$$
\begin{aligned}
\left\|a(s, \gamma) \gamma^{\prime}\right\|_{L^{\infty}} \leq & \left\|a(s, \gamma) \gamma^{\prime}\right\|_{L^{1}}+\|\alpha\|_{L^{2}}+ \\
& + \text { const } \int_{0}^{1}\left|\gamma^{\prime}\right| \mathrm{d} s+\text { const } \int_{0}^{1}\left|\gamma^{\prime}\right|^{2} \mathrm{~d} s+\text { const } .
\end{aligned}
$$

On the hand, by hypothesis (1.1)

$$
\nu\left|\gamma^{\prime}\right|^{2} \leq\left(a(s, \gamma) \gamma^{\prime}, \gamma^{\prime}\right) \leq\left|a(s, \gamma) \gamma^{\prime}\right|\left|\gamma^{\prime}\right|
$$

which implies

$$
\nu\left\|\gamma^{\prime}\right\|_{L^{\infty}} \leq\left\|a(s, \gamma) \gamma^{\prime}\right\|_{L^{\infty}}
$$

Thus, $\gamma^{\prime} \in L^{\infty}$ and

$$
\begin{aligned}
\left\|\gamma^{\prime}\right\|_{L^{\infty}} \leq & \frac{1}{\nu}\left(\left\|a(s, \gamma) \gamma^{\prime}\right\|_{L^{1}}+\|\alpha\|_{L^{2}}+\text { const } \int_{0}^{1}\left|\gamma^{\prime}\right| \mathrm{d} s+\right. \\
& \left.+ \text { const } \int_{0}^{1}\left|\gamma^{\prime}\right|^{2} \mathrm{~d} s+\text { const }\right) .
\end{aligned}
$$

By using (2.5.3), we have

$$
\begin{aligned}
\int_{0}^{1}\left|\gamma^{\prime}\right|\left(1+\left|\gamma^{\prime}\right|\right)|\delta| \mathrm{d} s & \leq\|\delta\|_{L^{2}}\left\|\gamma^{\prime}\right\|_{L^{2}}+\|\delta\|_{L^{2}}\left\|\gamma^{\prime}\right\|_{L^{4}}^{2} \leq \\
\leq & \|\delta\|_{L^{2}}\left\|\gamma^{\prime}\right\|_{L^{2}}+\|\delta\|_{L^{2}}\left\|\gamma^{\prime}\right\|_{L^{2}}\left\|\gamma^{\prime}\right\|_{L^{\infty}} \leq \\
\leq & \left\|\gamma^{\prime}\right\|_{L^{2}}\left(1+\left\|\gamma^{\prime}\right\|_{L^{\infty}}\right)\|\delta\|_{L^{2}} \leq \\
\leq & \left\|\gamma^{\prime}\right\|_{L^{2}}\left(1+\frac{1}{\nu}\left(\text { const }\left\|\gamma^{\prime}\right\|_{L^{1}}+\|\alpha\|_{L^{2}}+\right.\right. \\
& \left.\left.+ \text { const }\left\|\gamma^{\prime}\right\|_{L^{2}}^{2}+\text { const }\right)\right)\|\delta\|_{L^{2}}
\end{aligned}
$$




\section{Annamaria Canino}

Since,

$$
\frac{1}{2} \int_{0}^{1}\left|\frac{\partial a(s, \gamma)}{\partial q}\right||\delta|\left|\gamma^{\prime}\right|^{2} \mathrm{~d} s \leq \mathrm{const}\|\delta\|_{L^{2}}\left\|\gamma^{\prime}\right\|_{L^{4}}^{2}
$$

by (2.4.1) and (2.5.4), we have

$$
\begin{aligned}
& \left|\int_{0}^{1}\left(a(s, \gamma) \gamma^{\prime}, \delta^{\prime}\right)\right| \mathrm{d} s \leq \\
& \quad \leq\left[\|\alpha\|_{L^{2}}+\text { const }\left\|\gamma^{\prime}\right\|_{L^{2}}\left(1+\left\|\gamma^{\prime}\right\|_{L^{1}}+\|\alpha\|_{L^{2}}+\left\|\gamma^{\prime}\right\|_{L^{2}}^{2}\right)+\right. \\
& \quad+\text { const }]\|\delta\|_{L^{2}} \leq \\
& \quad \leq\left[\left(1+\text { const }\left\|\gamma^{\prime}\right\|_{L^{2}}\right)\|\alpha\|_{L^{2}}+\right. \\
& \left.\quad+\text { const }\left\|\gamma^{\prime}\right\|_{L^{2}}\left(1+\left\|\gamma^{\prime}\right\|_{L^{1}}+\left\|\gamma^{\prime}\right\|_{L^{2}}^{2}\right)+\text { const }\right]\|\delta\|_{L^{2}} .
\end{aligned}
$$

So, we can conclude that

$$
a(s, \gamma) \gamma^{\prime} \in W^{1,2}\left(0,1, \mathbb{R}^{n}\right) .
$$

Moreover, by (2.4.1), we deduce that

$$
\left.\left|\frac{\mathrm{d}}{\mathrm{d} s}\left(a(s, \gamma) \gamma^{\prime}\right)\right| \leq \operatorname{const}\left(1+|\alpha|+\left|\gamma^{\prime}\right|^{2}\right) \quad \text { a.e. } \quad \text { in }\right] 0,1[\text {. }
$$

It remains to prove that $\gamma^{\prime} \in W^{1,2}\left(0,1 ; \mathbb{R}^{n}\right)$. By hypothesis (1.1), we have

$$
\begin{aligned}
& \nu^{2} \frac{\left|\gamma^{\prime}\left(s_{2}\right)-\gamma^{\prime}\left(s_{1}\right)\right|^{2}}{\left|s_{2}-s_{1}\right|} \leq \\
& \quad \leq \frac{\left|a\left(s_{1}, \gamma\left(s_{1}\right)\right)\left(\gamma^{\prime}\left(s_{2}\right)-\gamma^{\prime}\left(s_{1}\right)\right)\right|^{2}}{\left|s_{2}-s_{1}\right|}=\frac{\mathfrak{N}}{\left|s_{2}-s_{1}\right|} \leq \\
& \quad \leq 2 \frac{\left|a\left(s_{2}, \gamma\left(s_{2}\right)\right) \gamma^{\prime}\left(s_{2}\right)-a\left(s_{1}, \gamma\left(s_{1}\right)\right) \gamma^{\prime}\left(s_{1}\right)\right|^{2}}{\left|s_{2}-s_{1}\right|}+ \\
& \quad+2 \frac{\left|a\left(s_{2}, \gamma\left(s_{2}\right)\right)-a\left(s_{1}, \gamma\left(s_{1}\right)\right)\right|\left|\gamma^{\prime}\left(s_{2}\right)\right|^{2}}{\left|s_{2}-s_{1}\right|}
\end{aligned}
$$

where

$$
\begin{aligned}
\mathfrak{N}= & \mid a\left(s_{2}, \gamma\left(s_{2}\right)\right) \gamma^{\prime}\left(s_{2}\right)-a\left(s_{1}, \gamma\left(s_{1}\right)\right) \gamma^{\prime}\left(s_{1}\right)+ \\
& -\left.\left[a\left(s_{2}, \gamma\left(s_{2}\right)\right)-a\left(s_{1}, \gamma\left(s_{1}\right)\right)\right] \gamma^{\prime}\left(s_{2}\right)\right|^{2} .
\end{aligned}
$$


Since $a(\gamma), a(s, \gamma) \gamma^{\prime} \in W^{1,2}\left(0,1 ; \mathbb{R}^{n}\right)$ and $\gamma^{\prime} \in L^{\infty}\left(0,1 ; \mathbb{R}^{n}\right)$, we deduce that

$$
\gamma^{\prime} \in W^{1,2}\left(0,1 ; \mathbb{R}^{n}\right)
$$

and

$$
\begin{aligned}
\left|\gamma^{\prime \prime}\right| & \leq \frac{1}{\nu}\left(\left|\frac{\mathrm{d}}{\mathrm{d} s}\left(a(s, \gamma) \gamma^{\prime}\right)\right|+\left|\frac{\mathrm{d}}{\mathrm{d} s}(a(s, \gamma))\right|\left|\gamma^{\prime}\right|\right) \leq \\
& \left.\leq \operatorname{const}\left(1+|\alpha|+\left|\gamma^{\prime}\right|^{2}\right) \quad \text { a.e. in }\right] 0,1[.
\end{aligned}
$$

If we set

$$
\tilde{\gamma}(s)= \begin{cases}\gamma\left(s+\frac{1}{2}\right) & 0 \leq s \leq \frac{1}{2} \\ \gamma\left(s-\frac{1}{2}\right) & \frac{1}{2} \leq s \leq 1\end{cases}
$$

it turns out that $\tilde{\gamma}$ also satisfies (2.4.1) with $a, V$ and $\alpha$ substituted by other suitable maps. It follows that

$$
\tilde{\gamma} \in W^{2,2}\left(0,1 ; \mathbb{R}^{n}\right),
$$

hence

$$
\gamma_{+}^{\prime}(0)=\gamma_{-}^{\prime}(1)
$$

Since $\partial^{-} I_{M}(\gamma(s))$ is a closed convex cone, to prove ii) is equivalent to prove that a.e. $\forall \eta \in\left(\partial^{-} I_{M}(\gamma(s))\right)^{-}$

$$
\left(\alpha+\frac{\mathrm{d}}{\mathrm{d} s}\left(a(s, \gamma) \gamma^{\prime}\right)+\nabla_{q} V(s, \gamma)-\frac{1}{2} \frac{\partial a(s, \gamma)}{\partial q}\left(\gamma^{\prime}, \gamma^{\prime}\right), \eta\right) \leq 0 .
$$

Let us define the following functions:

$$
\delta_{n}(s)=\rho_{n}\left(s-s_{0}\right) \eta, \quad \forall n \in \mathbb{N}
$$

where

$$
\left\{\begin{array}{l}
\left.s_{0} \in\right] 0,1\left[\text { is a Lebesgue point for } \alpha \text { and for } \frac{\mathrm{d}}{\mathrm{d} s}\left(a(s, \gamma) \gamma^{\prime}\right)\right. \\
\eta \in\left(\partial^{-} I_{M}\left(\gamma\left(s_{0}\right)\right)\right)^{-} ; \\
\rho_{n} \in C_{0}^{\infty}(\mathbb{R}), \quad \rho_{n} \geq 0, \quad \int_{0}^{1} \rho_{n} \mathrm{~d} s=1 \quad \text { and } \quad \text { supt } \rho_{n} \subset\left[-\frac{1}{n}, \frac{1}{n}\right] .
\end{array}\right.
$$




\section{Annamaria Canino}

Clearly, $\delta_{n} \in W_{0}^{1,2}\left(0,1 ; \mathbb{R}^{n}\right)$ and then by $(2.4 .1)$ we get

$$
\begin{aligned}
& -\left(\eta, \int_{0}^{1} \rho_{n}\left(s_{0}-s\right) \frac{\mathrm{d}}{\mathrm{d} s}\left(a(s, \gamma) \gamma^{\prime}\right) \mathrm{d} s\right) \geq \\
& \geq \int_{0}^{1} \rho_{n}\left(s_{0}-s\right)\left(\alpha(s), P_{\gamma} \eta\right)(s) \mathrm{d} s+ \\
& \quad-\operatorname{const} \int_{0}^{1} \rho_{n}\left(s_{0}-s\right)\left|\eta-P_{\gamma} \eta\right|\left|\gamma^{\prime}\right| \mathrm{d} s+ \\
& \quad-\operatorname{const} \int_{0}^{1} \rho_{n}\left(s_{0}-s\right)\left|\eta-P_{\gamma} \eta\right|\left|\gamma^{\prime}\right|^{2} \mathrm{~d} s+ \\
& \quad+\int_{0}^{1} \rho_{n}\left(s_{0}-s\right)\left(\nabla_{q} V(s, \gamma), P_{\gamma} \eta\right)(s) \mathrm{d} s+ \\
& \quad-\frac{1}{2} \int_{0}^{1} \rho_{n}\left(s_{0}-s\right)\left(\frac{\partial a(s, \gamma)}{\partial q}\left(P_{\gamma} \eta\right) \gamma^{\prime}, \gamma^{\prime}\right) \mathrm{d} s .
\end{aligned}
$$

By proposition $1.13, P_{\gamma} \delta$ is continuous at $s_{0}$, hence passing to the limit as $s \rightarrow s_{0}$ in (2.5.7), we obtain

$$
\begin{aligned}
- & \left(\eta, \frac{\mathrm{d}}{\mathrm{d} s}\left(a\left(s_{0}, \gamma\left(s_{0}\right)\right) \gamma^{\prime}\left(s_{0}\right)\right)\right) \geq \\
\geq & \left(\alpha\left(s_{0}\right),\left(P_{\gamma} \eta\right)\left(s_{0}\right)\right)-\text { const }\left|\eta-P_{\gamma} \eta\right|\left(s_{0}\right)\left|\gamma^{\prime}\right|^{2}\left(s_{0}\right)+ \\
& \quad-\text { const }\left|\eta-P_{\gamma} \eta\right|\left(s_{0}\right)\left|\gamma^{\prime}\right|\left(s_{0}\right)+\left(\nabla_{q} V\left(s_{0}, \gamma\left(s_{0}\right)\right), P_{\gamma} \eta\right)\left(s_{0}\right)+ \\
& -\frac{1}{2}\left(\frac{\partial a\left(s_{0}, \gamma\left(s_{0}\right)\right)}{\partial q}\left(P_{\gamma} \eta\right)\left(s_{0}\right) \gamma^{\prime}\left(s_{0}\right), \gamma^{\prime}\left(s_{0}\right)\right)= \\
= & \left(\alpha\left(s_{0}\right), \eta\left(s_{0}\right)\right)+\left(\nabla_{q} V\left(s_{0}, \gamma\left(s_{0}\right)\right), P_{\gamma} \eta\right)\left(s_{0}\right)+ \\
& \left.-\frac{1}{2}\left(\frac{\partial a\left(s_{0}, \gamma\left(s_{0}\right)\right)}{\partial q}\left(P_{\gamma} \eta\right)\left(s_{0}\right) \gamma^{\prime}\left(s_{0}\right), \gamma^{\prime}\left(s_{0}\right)\right) \text { a.e. in }\right] 0,1[.
\end{aligned}
$$

Finally, we are able to prove theorem 2.1.

Proof of theorem 2.1

As a direct consequence of lemmas 2.4 and 2.5, we get

$$
\gamma \in W^{2,2}\left(0,1 ; \mathbb{R}^{n}\right), \quad \gamma_{+}^{\prime}(0)=\gamma_{-}^{\prime}(1)
$$

and

$$
\left.\left|\gamma^{\prime \prime}(s)\right| \leq \text { const }\left(1+|\alpha(s)|+\left|\gamma^{\prime}(s)\right|^{2}\right) \quad \text { a.e. in }\right] 0,1[\text {. }
$$


If $0 \in \partial^{-} f(\gamma)$, it is evident that

$$
\gamma^{\prime \prime} \in L^{\infty}\left(0,1 ; \mathbb{R}^{n}\right)
$$

Now, let us prove two properties of $f$.

TheOREM 2.6. - The functional $f: L^{2}\left(0,1 ; \mathbb{R}^{n}\right) \rightarrow \mathbb{R} \cup\{+\infty\}$ is lower semicontinuous and there exists a continuous function $\theta: \mathbb{R} \rightarrow \mathbb{R}$ such that

$$
f(\gamma+\delta)-f(\gamma)-\int_{0}^{1}(\alpha, \delta) \mathrm{d} s \geq-\theta(f(\gamma))\left(1+\|\alpha\|_{L^{2}}^{2}\right)\|\delta\|_{L^{2}}^{2}
$$

whenever $\gamma, \gamma+\delta \in X$ and $\alpha \in \partial^{-} f(\gamma)$.

In particular, $f$ has a $\varphi$-monotone subdifferential of order two.

Proof. - Let us take a sequence $\left\{\gamma_{n}\right\}_{n} \subset X$ such that

$$
\lim _{n} \gamma_{n}=\gamma \text { in } L^{2}\left(0,1 ; \mathbb{R}^{n}\right) \text { and } f\left(\gamma_{n}\right) \leq c .
$$

In order to prove that $f$ is lower semicontinuous, it is enough to prove that $f(\gamma) \leq c$. Since,

$$
\frac{1}{2} \int_{0}^{1}\left(a\left(s, \gamma_{n}\right) \gamma_{n}^{\prime}, \gamma_{n}^{\prime}\right) \mathrm{d} s-\int_{0}^{1} V\left(s, \gamma_{n}\right) \mathrm{d} s \leq c
$$

and by hypothesis (1.1)

$$
\int_{0}^{1}\left(a\left(s, \gamma_{n}\right) \gamma_{n}^{\prime}, \gamma_{n}^{\prime}\right) \mathrm{d} s \geq \nu \int_{0}^{1}\left|\gamma_{n}^{\prime}\right|^{2} \mathrm{~d} s
$$

we can deduce that $\left\{\gamma_{n}^{\prime}\right\}_{n}$ is bounded in $L^{2}\left(0,1 ; \mathbb{R}^{n}\right)$ and thus, by the compactness of $M, \gamma_{n}$ weakly converges to $\gamma$ in $W^{1,2}\left(0,1 ; \mathbb{R}^{n}\right)$. Besides, $L$ is continuous in the three variables and convex in the third one, so it is weakly lower semicontinuous in $W^{1,2}\left(0,1 ; \mathbb{R}^{n}\right)$. This implies

$$
\begin{aligned}
& \frac{1}{2} \int_{0}^{1}\left(a(s, \gamma) \gamma^{\prime}, \gamma^{\prime}\right) \mathrm{d} s-\int_{0}^{1} V(s, \gamma) \mathrm{d} s \leq \\
& \quad \leq \liminf _{n} \frac{1}{2} \int_{0}^{1}\left(a\left(s, \gamma_{n}\right) \gamma_{n}^{\prime}, \gamma_{n}^{\prime}\right) \mathrm{d} s-\int_{0}^{1} V\left(s, \gamma_{n}\right) \mathrm{d} s \leq c .
\end{aligned}
$$

Moreover, $\left\{\gamma_{n}\right\}_{n}$ converges uniformly to $\gamma$. Since $M$ is closed, then $\gamma \in X$. 
Annamaria Canine

Now, let us take $\gamma \in X \cap W^{2,2}\left(0,1 ; \mathbb{R}^{n}\right)$, with $\gamma_{+}^{\prime}(0)=\gamma_{-}^{\prime}(1)$ and $\alpha \in \partial^{-} f(\gamma)$. Let $\delta \in W^{1,2}\left(0,1 ; \mathbb{R}^{n}\right)$ with $\delta(0)=\delta(1)$ be such that $\gamma+\delta \in X$. Then, by Taylor's formula, we have:

$$
\begin{aligned}
f(\gamma+ & \delta)-f(\gamma)-\int_{0}^{1}(\alpha, \delta) \mathrm{d} s= \\
= & \frac{1}{2} \int_{0}^{1}\left(a(s, \gamma+\delta)\left(\gamma^{\prime}+\delta^{\prime}\right),\left(\gamma^{\prime}+\delta^{\prime}\right)\right) \mathrm{d} s-\frac{1}{2} \int_{0}^{1}\left(a(s, \gamma) \gamma^{\prime}, \gamma^{\prime}\right) \mathrm{d} s+ \\
& -\int_{0}^{1} V(s, \gamma+\delta) \mathrm{d} s+\int_{0}^{1} V(s, \gamma) \mathrm{d} s-\int_{0}^{1}(\alpha, \delta) \mathrm{d} s= \\
= & \frac{1}{2} \int_{0}^{1}\left(\frac{\partial a(s, \gamma)}{\partial q}(\delta) \gamma^{\prime}, \gamma^{\prime}\right) \mathrm{d} s-\int_{0}^{1}\left(\nabla_{q} V(s, \gamma), \delta\right) \mathrm{d} s+ \\
& +\int_{0}^{1}\left(a(s, \gamma) \gamma^{\prime}, \delta^{\prime}\right) \mathrm{d} s-\int_{0}^{1}(\alpha, \delta) \mathrm{d} s+ \\
& +\frac{1}{4} \int_{0}^{1}\left(\frac{\partial^{2} a(s, \tilde{\gamma})}{\partial q^{2}}(\delta)^{2} \gamma^{\prime}, \gamma^{\prime}\right) \mathrm{d} s-\frac{1}{2} \int_{0}^{1}\left(\nabla_{q q}^{2} V(s, \gamma) \delta, \delta\right) \mathrm{d} s+ \\
& +\frac{1}{4} \int_{0}^{1}\left(\frac{\partial a(s, \tilde{\gamma})}{\partial q}(\delta), \delta^{\prime}\right) \mathrm{d} s+\frac{1}{4} \int_{0}^{1}\left(a(s, \gamma) \delta^{\prime}, \delta^{\prime}\right) \mathrm{d} s
\end{aligned}
$$

where $\tilde{\gamma}=\gamma+t \delta$ for some $t=t(s) \in] 0,1[$. Reordering terms, by hypothesis (1.1), we get:

$$
\begin{aligned}
f(\gamma+ & \delta)-f(\gamma)-\int_{0}^{1}(\alpha, \delta) \mathrm{d} s \geq \\
\geq & \frac{1}{2} \int_{0}^{1}\left(\frac{\partial a(s, \gamma)}{\partial q}\left(\gamma^{\prime}, \gamma^{\prime}\right)-\nabla_{q} V(s, \gamma)-\frac{\mathrm{d}}{\mathrm{d} s}\left(a(s, \gamma) \gamma^{\prime}\right)-\alpha, \delta\right) \mathrm{d} s+ \\
& -\left(\frac{1}{4}\left\|\frac{\delta^{2} a(s, \gamma)}{\partial q^{2}}\right\|_{L^{\infty}}\left\|\gamma^{\prime}\right\|_{L^{2}}^{2}+\frac{1}{2}\left\|\nabla_{q q} V(s, \gamma)\right\|_{L^{\infty}}\right)\|\delta\|_{L^{\infty}}^{2}+ \\
& -\frac{1}{4}\left\|\frac{\delta a(s, \gamma)}{\partial q}\right\|_{L^{\infty}}\|\delta\|_{L^{2}}\left\|\delta^{\prime}\right\|_{L^{2}}+\frac{\nu}{2}\left\|\delta^{\prime}\right\|_{L^{2}}^{2} .
\end{aligned}
$$

Thus, by $p$-convexity of $M$, we get:

$$
\begin{aligned}
f(\gamma+\delta)-f(\gamma)-\int_{0}^{1}(\alpha, \delta) \mathrm{d} s \geq \\
\geq-\left(\frac{1}{2}\left\|\frac{\partial a(s, \gamma)}{\partial q}\right\|_{L^{\infty}}\left\|\gamma^{\prime}\right\|_{L^{2}}+\left\|\nabla_{q} V(s, \gamma)\right\|+\right. \\
\left.\quad+\left\|\frac{\mathrm{d}}{\mathrm{d} s}(a(s, \gamma)) \gamma^{\prime}+a(s, \gamma) \gamma^{\prime \prime}\right\|_{L^{1}}+\|\alpha\|_{L^{1}}\right)\|\delta\|_{L^{\infty}}+
\end{aligned}
$$

-56 - 
Periodic solutions of quadratic Lagrangian systems on $p$-convex sets

$$
\begin{aligned}
& - \text { const }\left\|\gamma^{\prime}\right\|_{L^{2}}^{2}\|\delta\|_{L^{\infty}}^{2}-\text { const }\|\delta\|_{L^{\infty}}\left\|\delta^{\prime}\right\|_{L^{2}}+\frac{\nu}{2}\left\|\delta^{\prime}\right\|_{L^{2}}^{2} \geq \\
& \geq-\operatorname{const}\left(\left\|\gamma^{\prime \prime}\right\|_{L^{1}}+\|\alpha\|_{L^{1}}\right)\|\delta\|_{L^{\infty}}-\text { const }\left\|\gamma^{\prime}\right\|_{L^{2}}^{2}\|\delta\|_{L^{\infty}}^{2}+ \\
& - \text { const }\|\delta\|_{L^{\infty}}\left\|\delta^{\prime}\right\|_{L^{2}}+\frac{\nu}{2}\left\|\delta^{\prime}\right\|_{L^{2}}^{2} .
\end{aligned}
$$

Using the interpolation inequality:

$$
\|\delta\|_{L^{\infty}}^{2} \leq\|\delta\|_{L^{2}}^{2}+2\|\delta\|_{L^{2}}\left\|\delta^{\prime}\right\|_{L^{2}}
$$

we obtain:

$$
\begin{aligned}
f(\gamma+\delta)-f(\gamma)-\int_{0}^{1}(\alpha, \delta) \mathrm{d} s \geq \\
\geq-\operatorname{const}\left(\left\|\gamma^{\prime \prime}\right\|_{L^{1}}+\|\alpha\|_{L^{1}}\right)\left(\|\delta\|_{L^{2}}^{2}+2\|\delta\|_{L^{2}}\left\|\delta^{\prime}\right\|_{L^{2}}\right)+ \\
\quad-\operatorname{const}\left\|\gamma^{\prime}\right\|_{L^{2}}\left(\|\delta\|_{L^{2}}^{2}+2\|\delta\|_{L^{2}}\left\|\delta^{\prime}\right\|_{L^{2}}\right)+ \\
\quad-\operatorname{const}\left(\|\delta\|_{L^{2}}\left\|\delta^{\prime}\right\|_{L^{2}}+\|\delta\|_{L^{2}}^{1 / 2}\left\|\delta^{\prime}\right\|_{L^{2}}^{3 / 2}\right)+\frac{\nu}{2}\left\|\delta^{\prime}\right\|_{L^{2}}^{2}
\end{aligned}
$$

Thus, by theorem 2.1 and hypothesis 1.1 , we are able to conclude that:

$$
f(\gamma+\delta)-f(\gamma)-\int_{0}^{1}(\alpha, \delta) \mathrm{d} s \geq-\theta(f(\gamma))\left(1+\|\alpha\|^{2}\right)\|\delta\|_{L^{2}}^{2}
$$

ThEOREM 2.7. - Let us consider $\alpha \in L^{2}\left(0,1 ; \mathbb{R}^{n}\right)$ and $\gamma \in X \cap$ $W^{2,2}\left(0,1 ; \mathbb{R}^{n}\right)$ with $\gamma^{+}(0)=\gamma^{-}(1)$. Then $\alpha \in \partial^{-} f(\gamma)$ if and only if

$$
\alpha(s)+\frac{\mathrm{d}}{\mathrm{d} s}\left(a(s, \gamma) \gamma^{\prime}\right)+\nabla_{q} V(s, \gamma)-\frac{1}{2} \frac{\partial a(s, \gamma)}{\partial q}\left(\gamma^{\prime}, \gamma^{\prime}\right) \in \partial^{-} I_{M}(\gamma(s)) \text { a.e. }
$$

Proof. - If $\alpha \in \partial^{-} f(\gamma)$, we have the thesis by lemmas 2.4 and 2.5. Viceversa, if

$$
\alpha(s)+\frac{\mathrm{d}}{\mathrm{d} s}\left(a(s, \gamma) \gamma^{\prime}\right)+\nabla_{q} V(s, \gamma)-\frac{1}{2} \frac{\partial a(s, \gamma)}{\partial q}\left(\gamma^{\prime}, \gamma^{\prime}\right) \in \partial^{-} I_{M}(\gamma(s)) \text { a.e. }
$$

the proof of theorem 2.6 shows that $\alpha \in \partial^{-} f(\gamma)$.

Finally, we can state the already quoted characterization. 
THEOREM 2.8. - Let us consider $\gamma \in X$. Then $0 \in \partial^{-} f(\gamma)$ if and only if $\gamma \in W^{2, \infty}, \gamma_{+}^{\prime}(0)=\gamma_{-}^{\prime}(1)$ and

$$
\alpha(s)+\frac{\mathrm{d}}{\mathrm{d} s}\left(a(s, \gamma) \gamma^{\prime}\right)+\nabla_{q} V(s, \gamma)-\frac{1}{2} \frac{\partial a(s, \gamma)}{\partial q}\left(\gamma^{\prime}, \gamma^{\prime}\right) \in \partial^{-} I_{M}(\gamma(s)) \text { a.e. }
$$

Proof. - If $0 \in \partial^{-} f(\gamma)$, from theorem $2.1 \gamma \in W^{2, \infty}\left(0,1 ; \mathbb{R}^{n}\right)$ and $\gamma^{+}(0)=\gamma^{-}(1)$. Moreover from theorem 2.7

$$
\alpha(s)+\frac{\mathrm{d}}{\mathrm{d} s}\left(a(s, \gamma) \gamma^{\prime}\right)+\nabla_{q} V(s, \gamma)-\frac{1}{2} \frac{\partial a(s, \gamma)}{\partial q}\left(\gamma^{\prime}, \gamma^{\prime}\right) \in \partial^{-} I_{M}(\gamma(s)) \text { a.e. }
$$

Viceversa, it is enough to apply theorem 2.7 with $\alpha=0$.

\section{The category of the space of the admissible paths}

After theorem 2.8, our goal is to prove the existence of infinitely many lower critical points for $f$ on $X$ by means to theorem 1.7. Therefore, let us investigate the topological properties of $X$.

If $Y$ is a topological space, we will denote by $\Lambda(Y)$ the free loop space of $Y$.

Let us recall that in [5], using results contained in [12], [13] and in [19], it is proved the following theorem.

TheOREM 3.1.- (see theorem 3.3 in [5]) Let $A$ be an open subset of $\mathbb{R}^{n}$, connected and non-contractible in itself. Moreover, let us suppose that either

i) $\pi_{1}(A)$ has infinitely many conjugacy classes or

ii) $\pi_{1}(A)$ has a finite number of elements.

Then cat $\Lambda(A)=+\infty$.

Now, let us consider $X$ endowed with the $W^{1,2}$-topology and the space

$$
\Lambda(M)=\{\gamma \mid[0,1] \rightarrow M, \gamma \text { is continuous and } \gamma(0)=\gamma(1)\}
$$

endowed with the uniform topology. 
Periodic solutions of quadratic Lagrangian systems on p-convex sets

Theorem 3.2. - (see theorem 4.5 in [4]) The inclusion map $i: X \rightarrow$ $\Lambda(M)$ is a homotopy equivalence.

Now, we are to able evaluate the category of $X$.

THEOREM 3.3. - Let $M \subset \mathbb{R}^{n}$ be a connected, non-contractible in itself, compact p-convex set. Let us suppose that either

i) $\pi_{1}(M)$ has infinitely many conjugacy classes

or

ii) $\pi_{1}(M)$ has a finite number of elements.

Then $\operatorname{cat}(X)=+\infty$.

Proof. - Let us consider $A$, the open subset of $\mathbb{R}^{n}$ defined in Remark 1.10. Clearly, $M$ is a deformation retract of it. Then, $A$ is homotopically equivalent to $M$ and $\Lambda(A)$ is homotopically equivalent to $\Lambda(M)$. By applying theorems 3.1 and 3.2 , the proof is over.

Finally we are able to prove the main theorem.

\section{Proof of theorem 1.4}

We want to apply theorem 1.7. Let us consider the functional $f$ defined in section 2. By hypothesis (1.1) and theorem 2.6, $f$ is a lower semicontinuous function, bounded below and it has a $\varphi$-monotone subdifferential of order two.

Moreover, let us observe that $D(f)=X$ and that $d^{*}$ induces the $W^{1,2}$. topology on $X$. By theorem 3.3, $\operatorname{cat}\left(D(f), d^{*}\right)=+\infty$.

Now, we will consider a sequence $\left\{\gamma_{h}\right\}_{h} \subset D\left(\partial^{-} f\right)$ with $\sup _{h} f\left(\gamma_{h}\right)<$ $+\infty$ and $\lim _{h} \operatorname{grad}^{-} f\left(\gamma_{h}\right)=0$. Since $M$ is compact, $\left\{\gamma_{h}\right\}_{h}$ is bounded in $L^{2}\left(0,1 ; \mathbb{R}^{n}\right)$. But also $\left\{\gamma_{h}^{\prime}\right\}_{h}$ is bounded in $L^{2}\left(0,1 ; \mathbb{R}^{n}\right)$. Thus, by Rellich's theorem, $\left\{\gamma_{h}\right\}_{h}$ has a subsequence converging in $L^{2}\left(0,1 ; \mathbb{R}^{n}\right)$.

So, applying theorem 1.7 and theorem 2.8 , the thesis follows.

\section{References}

[1] Benci (V.) . - Periodic solutions of Lagrangian systems on a compact manifold, J. Differential Equations 63 (1986), pp. 135-161.

[2] Canino (A.) .- On p-convex sets and geodesics, J. Differential Equations 75 (1988), pp. 118-157. 


\section{Annamaria Canino}

[3] Canino (A.) . - Existence of a closed geodesic on p-convex sets, Ann. Inst. H. Poincaré, Anal. Non Linéaire 5 (1988), pp. 501-518.

[4] Canino (A.). - Local properties of geodesics on p-convex sets, Ann. Mat. Pura Appl., (in press).

[5] Canino (A.) .- Periodic solutions of Lagrangian systems on manifolds with boundary, Nonlinear Anal., 16 (1991), pp. 567-586.

[6] Сhobanov (G.), Marino (A.) and Scolozzi (D.) .- Evolution equation for the eigenvalue problem for the Laplace operator with respect to an obstacle, Rend. Accad. Naz. Sci. XL Mem. Mat. 5, (in press).

[7] Сhobanov (G.), Marino (A.) and Scolozzi (D.) - - Multiplicity of eigenvalues for the Laplace operator with respect to an obstacle and non tangency conditions, Nonlinear Anal. 15 (1990), pp. 199-215.

[8] De Giorgi (E.), Degiovani (M.), Marino (A.) and Tosques (M.) .Evolution equations for a class of nonlinear operators,

Atti Accad. Naz. Lincei Rend. Cl. Sci. Fis. Mat. Natur. (8) 75 (1983), pp. 1-8.

[9] De Giorgi (E.), Marino (A.) and Tosques (M.) . - Problemi di evoluzione in spazi metrici e curve di massima pendenza,

Atti Accad. Naz. Lincei Rend. Cl. Sci. Fis. Mat. Natur. (8) 68 (1980), pp. 180-187.

[10] Degiovann (M.) - - Homotopical properties of a class of nonsmooth functions Ann. Mat. Pura Appl. (4) 156 (1990), pp. 37-71.

[11] Degiovann (M.), Marino (A.) and Tosques (M.) .- Evolution equations with lack of convexity, Nonlinear Anal. 9 (1985), pp. 1401-1443.

[12] Fadell (E.) and Husseini (S.) .- A note on the category of the free loop space, Proc. Amer. Math. Soc. 107 (1989), pp. 527-536.

[13] FAdell (E.) and Husseini (S.) . - Extending Serre's theorem on the category of loop spaces, Preprint, 1990.

[14] Marino (A.) and Scolozzi (D.) - - Geodetiche con ostacolo, Boll. Un. Mat. Ital. B(6) 2 (1983), pp. 1-31.

[15] Scolozzi (D.) .- Esistenza e molteplicità di geodetiche con ostacolo e con estremi variabili, Ricerche Mat. 33 (1984), pp. 171-201.

[16] SColozzi (D.) .- Un teorema di esistenza di una geodetica chiusa su variatà con bordo, Boll. Un. Mat. Ital. A(6) 4 (1985), pp. 451-547.

[17] Scolozzi (D.) . - Molteplicità di curve con ostacolo e stazionarie per una classe di funzionali non regolari, Preprint $n^{\circ}$ 69, Dip. Mat. Pisa, 1984.

[18] Scolozzi (D.) .- Esistenza di una curva chiusa stazionaria e con ostacolo, Preprint $\mathrm{n}^{\circ}$ 70, Dip. Mat. Pisa, 1984.

[19] SerRe (J.P.) .- Homologie singulière des espaces fibrés, Ann. of Math. 54 (1951), pp. 425-505. 